\title{
Prediction Model of Interval Grey Numbers with a Real Parameter and Its Application
}

\author{
Bo Zeng, ${ }^{1,2}$ Chuan Li, ${ }^{2}$ Xue-Yu Zhou, ${ }^{2}$ and Xian-Jun Long ${ }^{3}$ \\ ${ }^{1}$ College of Business Planning, Chongqing Technology and Business University, Chongqing 400067, China \\ ${ }^{2}$ Chongqing Key Laboratory of Electronic \& Supply Chain System, Chongqing 400067, China \\ ${ }^{3}$ College of Mathematics and Statistics, Chongqing Technology and Business University, Chongqing 400067, China
}

Correspondence should be addressed to Chuan Li; lcctbu@126.com

Received 5 February 2014; Accepted 31 May 2014; Published 18 August 2014

Academic Editor: Jianqing Chen

Copyright (C) 2014 Bo Zeng et al. This is an open access article distributed under the Creative Commons Attribution License, which permits unrestricted use, distribution, and reproduction in any medium, provided the original work is properly cited.

\begin{abstract}
Grey prediction models have become common methods which are widely employed to solve the problems with "small examples and poor information." However, modeling objects of existing grey prediction models are limited to the homogenous data sequences which only contain the same data type. This paper studies the methodology of building prediction models of interval grey numbers that are grey heterogeneous data sequence, with a real parameter. Firstly, the position of the real parameter in an interval grey number sequence is discussed, and the real number is expanded into an interval grey number by adopting the method of grey generation. On this basis, a prediction model of interval grey number with a real parameter is deduced and built. Finally, this novel model is successfully applied to forecast the concentration of organic pollutant DDT in the atmosphere. The analysis and research results in this paper extend the object of grey prediction from homogenous data sequence to grey heterogeneous data sequence. Those research findings are of positive significance in terms of enriching and improving the theory system of grey prediction models.
\end{abstract}

\section{Introduction}

The grey system theory, established based on "grey box" developed by Julong Deng in 1982, is a new methodology that focuses on the study of problems involving small samples and poor information [1, 2]. It deals with partially known information through generating, excavating, and extracting useful information from what is available $[3,4]$. Therefore, systems' operational behaviors and their laws of evolution can be correctly described and effectively monitored [5]. A grey prediction model is one of the most important parts of grey system theory, and it has been employed in many fields, such as industry [6,7], agriculture [8], environment [9], and military [10]. According to the needs of solving practical problems, scholars conduct a lot of researches about the extension and optimization of grey prediction models in the appliance process. Those research findings are mainly concentrated in five aspects as follows.

(a) Study on the preprocessing methods of modeling sequences. It mainly includes improving the smoothness of sequence by function transformations [11] and weakening the effects of shocking disturbances to model structures through buffer operators [12]. Such measures can improve accuracy of models.

(b) Study on the optimization methods of grey prediction model parameters. It is mainly through mathematical methods to optimize initial values [13] and background values [14] of grey models, and this can improve the modeling parameters (that is, $a$ and $b$ ) of grey models.

(c) Study on modeling mechanisms of grey prediction models. It mainly includes researching on methods of grey generation of modeling sequence [15], model 
stability and ill-conditioned modeling conditions [16], and application ranges of grey prediction models [17].

(d) Study on some novel grey prediction model, such as discrete grey prediction model, abbreviated DGM $(1,1)$ model [18], nonequidistance grey prediction model, and abbreviated NGM $(1,1)$ model [19].

Other related studies. They include combining grey prediction models with other models or methods [20], for example, the Markov model or support vector machine [21, 22], to improve the accuracy of grey models, studying on the error check methods of grey prediction models.

The modeling objects of inchoate researches and applications of grey prediction models are mainly based on real numbers. However, because of the complexity of study objects or the limits of technical means and people's reason, the information in the real world is often shown in the form of grey numbers, such as environmental monitoring data may be an interval grey number which is located in a specific range (e.g., $\otimes \in[10,20])$ or the data is a discrete grey number which has some uncertain value (e.g., $\otimes \in\{10,15,18,22\}$ ). Provided this situation, it is not feasible to employ the traditional modeling methods based on real numbers in order to build grey prediction models with grey uncertainty characteristics modeling data sets. In order to tackle modeling problems of grey uncertain information, some novel concepts, such as grey band, grey layer, and standard discrete grey number, are proposed in literature $[23,24]$. In this research, different approaches are conducted in the study of modeling methods of interval and discrete grey number. This leads to a methodology which could expand modeling objects from real numbers to grey numbers efficiently.

Based on the above point of view, the grey prediction modeling objects could be categorized into the following: (i) sequence of real number, (ii) sequence of interval grey number, and (iii) sequence of discrete grey number. Each of these modeling object categories contains only one data type; they are all real numbers, interval grey numbers, or discrete grey numbers. However, there is a mixed data type of the modeling objects elements existing when the modeling process is employed into practical applications. For instance, in the sequence $S=(\{10,15,18,22\}, 25,[21,32],[25,34], 36)$, the first element " $\{10,15,18,22\}$ " of sequence $S$ is a discrete grey number, the second one " 25 " is a real number, the third one " $[21,32]$ " is an interval grey number, and so forth. Such sequences are named the heterogeneous data sequences. Under such circumstance, further research focused on how to build a grey prediction model based on heterogeneous data sequences will be investigated.

In this paper, the study on the prediction model of interval grey numbers with a real parameter will be conducted. In the target model, a grey heterogeneous modeling data sequence is adopted as the modeling objects element. Such modeling objects contain some interval grey numbers and one real number. In the modeling process, the real number is expanded into an interval grey number by adopting the method of traditional grey prediction model based on real numbers. Alternatively, the method of generation of nonadjacent neighbor mean could also bring the same process. Subsequently, the grey prediction models of interval grey number sequence and kernel sequence are built based on the authors' former research findings. Such models aim to forecast future of interval grey number and kernels.

Only the monogenesis data sequence can be simulated or forecasted by existing grey system models. Hence, all data of the modeling sequence should be within the same data type. This means that the existing grey models are invalid when dealing with two or more different types of the data in modeling sequence (i.e., grey heterogeneous data sequence). For this reason, a novel prediction model of interval grey numbers with a real parameter is proposed in this paper. The present work extends the modeling object from the monogenesis data sequence to the heterogeneous one. The application scope of the grey prediction models is expanded, such that it enriches the theory system of the grey prediction model with a positive significance.

The rest of the paper is organized as follows. In Section 2, the fundamentals of $\operatorname{DGM}(1,1)$ model and $\operatorname{IGM}(1,1)$ model are introduced. Section 3 studies the modeling methods of the novel grey prediction model of interval grey numbers with a real parameter, $\operatorname{IGNPM}\left(1,1, \phi_{m}\right)$ for short. In Section 4 , the relationships between $\operatorname{IGNPM}\left(1,1, \phi_{m}\right)$ model and other models are analyzed. Next, we employ IGNPM (1, $\left.1, \phi_{m}\right)$ model to forecast the changed trend of the concentration of DDT in atmosphere in Section 5. In Section 6, other contents associated with this paper are discussed. Finally, Section 7 concludes the paper and presents the future work.

\section{Preliminaries}

\section{1. $\operatorname{DGM}(1,1)$ Model}

Definition 1. Assume that

$$
X^{(0)}=\left(x^{(0)}(1), x^{(0)}(2), \ldots, x^{(0)}(n)\right)
$$

is a sequence, where

$$
x^{(0)}(k) \geq 0, \quad k=1,2, \ldots, n,
$$

and $X^{(1)}$ is the 1-AGO sequence of $X^{(0)}$ as follows:

$$
X^{(1)}=\left(x^{(1)}(1), x^{(1)}(2), \ldots, x^{(1)}(n)\right) \text {, }
$$

where

$$
\begin{gathered}
X^{(1)}(k)=\sum_{i=1}^{k} x^{(0)}(i), \\
x^{(1)}(k+1)=\beta_{1} x^{(1)}(k)+\beta_{2} .
\end{gathered}
$$

Then, formula (4) is known as a $\operatorname{DGM}(1,1)$ model or the discrete form of a GM $(1,1)$ model [18]. 
Theorem 2. If $\widehat{\beta}=\left(\beta_{1}, \beta_{2}\right)^{T}$ is a sequence parameter and

$$
Y=\left[\begin{array}{c}
x^{(1)}(2) \\
x^{(1)}(3) \\
\vdots \\
x^{(1)}(n)
\end{array}\right], \quad B=\left[\begin{array}{cc}
x^{(1)}(1) & 1 \\
x^{(1)}(2) & 1 \\
\vdots & \vdots \\
x^{(1)}(n-1) & 1
\end{array}\right],
$$

then the least square estimate sequence of grey differential formula (4) satisfies $\widehat{\beta}=\left(B^{T} B\right)^{-1} B^{T} Y$.

Theorem 3. If $B, Y, \widehat{\beta}$ are as stated in Theorem 2 and $\widehat{\beta}=\left[\beta_{1}\right.$, $\left.\beta_{2}\right]=\left(B^{T} B\right)^{-1} B^{Y}$, then we can obtain the following formulas.

(1) Let $x^{(1)}(1)=x^{(0)}(1)$, then

$$
\begin{array}{r}
\hat{x}^{(1)}(k+1)=\beta_{1}^{k} x^{(0)}(1)+\frac{1-\beta_{1}^{k}}{1-\beta_{1}} \beta_{2} ; \\
k=1,2, \ldots, n-1 .
\end{array}
$$

(2) The restored values $\widehat{x}^{(0)}(k+1)$ can be calculated as following:

$$
\begin{array}{r}
\widehat{x}^{(1)}(k+1)=\beta_{1}^{k}\left(x^{(0)}(1)-\frac{\beta_{2}}{1-\beta_{1}}\right)+\frac{\beta_{2}}{1-\beta_{1}} ; \\
k=1,2, \ldots, n-1 .
\end{array}
$$

Note. Proofs of Theorems 2 and 3 are omitted here, and the detailed information can be found in literature [18].

\subsection{Prediction Model of Interval Grey Number \\ (IGM $(1,1)$ Model)}

Definition 4. A grey number with both a lower limit $a_{m}$ and an upper limit $b_{m}$ is called an interval grey number, denoted as $\otimes\left(t_{m}\right) \in\left[a_{m}, b_{m}\right]$, where $b_{m} \geq a_{m}$. A sequence which consists of interval grey number $\otimes\left(t_{k}\right) \in\left[a_{k}, b_{k}\right](k=1,2, \ldots, n)$ is called an interval grey number sequence, denoted as $X(\otimes)=$ $\left(\otimes\left(t_{1}\right), \otimes\left(t_{2}\right), \ldots, \otimes\left(t_{n}\right)\right)$.

Definition 5. A sequence which consists of all those lower limits of interval grey numbers in $X(\otimes)$ is called the lower limit sequence of $X(\otimes)$, denoted as $X_{a}=\left(a_{1}, a_{2}, \ldots\right.$, $\left.a_{n}\right)$, where $X(\otimes)$ is an interval grey number defined in Definition 4. Similarly, one uses $X_{b}=\left(b_{1}, b_{2}, \ldots, b_{n}\right)$ to denote the upper limit sequence of $X(\otimes)$.

Assume that $X(\otimes)$ is an interval grey number sequence, that is

$$
X(\otimes)=\left(\otimes\left(t_{1}\right), \otimes\left(t_{2}\right), \ldots, \otimes\left(t_{n}\right)\right),
$$

where

$$
\Delta t_{k}=t_{k}-t_{k-1}=1, \quad \otimes\left(t_{k}\right) \in\left[a_{k}, b_{k}\right], k=1,2, \ldots, n .
$$

According to literature [25], $X(\otimes)$ can be equivalently transformed into two real number sequences $S$ and $W$; that is,

$$
\begin{gathered}
S=\left(s\left(t_{1}\right), s\left(t_{2}\right), \ldots, s\left(t_{n-1}\right)\right) \\
W=\left(w\left(t_{1}\right), w\left(t_{2}\right), \ldots, w\left(t_{n-1}\right)\right),
\end{gathered}
$$

where

$$
\begin{array}{r}
s\left(t_{p}\right)=\frac{\left(b_{p}-a_{p}\right)+\left(b_{p+1}-a_{p+1}\right)}{2}, \\
p=1,2, \ldots, n-1, \\
w\left(t_{p}\right)=\frac{\left(a_{p}+b_{p}\right)+\left(a_{p+1}+b_{p+1}\right)}{4}, \\
p=1,2, \ldots, n-1 .
\end{array}
$$

It has been proved in the literature [25] that an interval grey number sequence $X(\otimes)$ and its transformed sequences (that is, $S$ and $W$ ) have the same amount of information. In this paper, we will adopt this result. Herein, we omit the proof. By building prediction DGM $(1,1)$ models of sequences $S$ and $W$, respectively, the grey prediction model based on interval grey number sequence $X(\otimes)$ is developed as follows:

$$
\begin{aligned}
& \widehat{\otimes}\left(t_{k}\right) \in\left[\widehat{a}_{k}, \widehat{b}_{k}\right] \Longleftarrow \\
& \left\{\begin{array}{c}
\widehat{a}_{k}=\left(\left(F_{w} \times \alpha_{1}^{k-3}\left[1-\left(-\alpha_{1}^{-1}\right)^{k-2}\right]\right.\right. \\
\left.-F_{s} \times \beta_{1}^{k-3}\left[1-\left(-\beta_{1}^{-1}\right)^{k-2}\right]\right) \\
\left.\times(2)^{-1}\right)+(-1)^{k} a_{2}, \\
\widehat{b}_{k}=\left(\left(F_{s} \times \beta_{1}^{k-3}\left[1-\left(-\beta_{1}^{-1}\right)^{k-2}\right]\right.\right. \\
\left.+F_{w} \times \alpha_{1}^{k-3}\left[1-\left(-\alpha_{1}^{-1}\right)^{k-2}\right]\right) \\
\left.\times(2)^{-1}\right)+(-1)^{k} b_{2},
\end{array}\right.
\end{aligned}
$$

where

$$
\begin{aligned}
& F_{s}=\frac{2\left[s\left(t_{1}\right) \times\left(\beta_{1}-1\right)+\beta_{2}\right]}{1+\beta_{1}^{-1}}, \\
& F_{w}=\frac{4\left[w\left(t_{1}\right) \times\left(\alpha_{1}-1\right)+\alpha_{2}\right]}{1+\alpha_{1}^{-1}} .
\end{aligned}
$$

The derivation process of the details and the meaning of parameters $\alpha_{1}, \alpha_{2}, \beta_{1}$, and $\beta_{2}$ can be inferred to literature [25].

\section{Modeling Process}

Definition 6. If an interval grey number sequence $X(\otimes)=$ $\left(\otimes\left(t_{1}\right), \otimes\left(t_{2}\right), \ldots, \otimes\left(t_{n}\right)\right)$ that is defined in Definition 1 has one and only one element $\otimes\left(t_{m}\right)$, where $\otimes\left(t_{m}\right)$ satisfies the constrain of $\otimes\left(t_{m}\right) \in\left[a_{m}, b_{m}\right]$, then $X(\otimes)$ is named an interval grey number sequence with a real number. $\otimes\left(t_{m}\right)$ is called the real number parameter of sequence $X(\otimes)$, denoted as $\phi_{m}$.

Definition 7. An interval grey number sequence with a real number can be divided into three types according to the location of $\phi_{m}$ in $X(\otimes)$ as follows:

(i) when $m=1, X(\otimes)$ is named the first element real parameter of interval grey number sequence, as shown in Figure 1(a); 


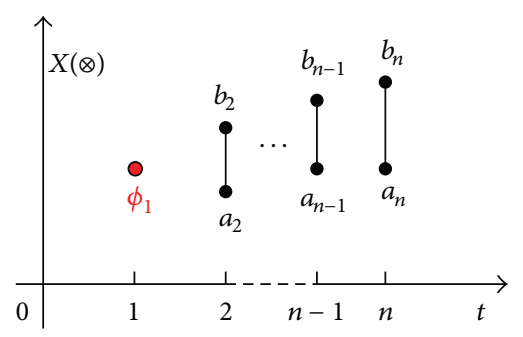

(a)

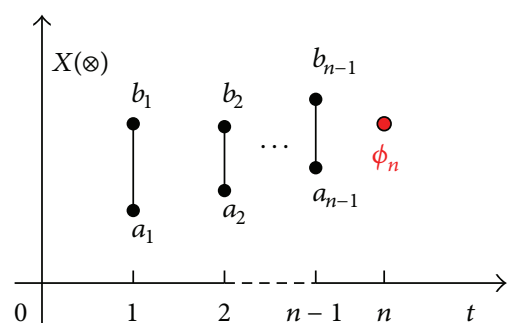

(b)

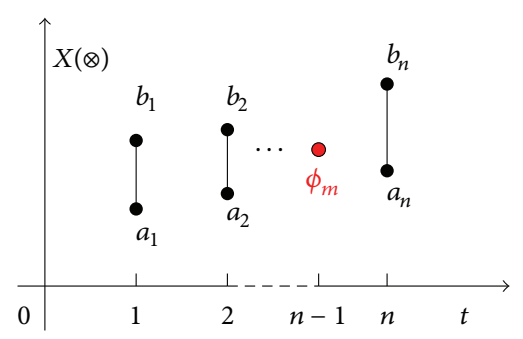

(c)

FIGURE 1: Three forms of interval grey number sequence with a real number.

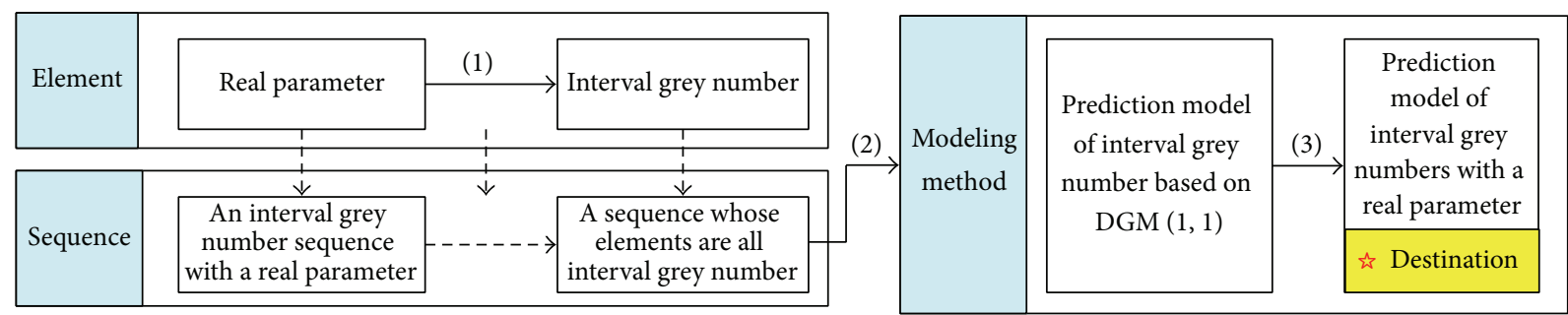

FIGURE 2: Process of modeling of interval grey numbers with a real parameter.

(ii) when $m=n, X(\otimes)$ is named the last element real parameter of interval grey number sequence, as shown in Figure 1(b);

(iii) when $m=2,3, \ldots, n-1, X(\otimes)$ is named the middle element parameter of interval grey number sequence, as shown in Figure 1(c).

According to the preknowledge in Section 2 of this paper, an interval grey number sequence $X(\otimes)$ can be transformed into two real number sequences $S$ and $W$ only when the elements of $X(\otimes)$ are all interval grey numbers. Subsequently, a grey prediction model of interval grey number based on $\operatorname{DGM}(1,1)$ model could be developed. On the other hand, it is not feasible to develop grey prediction models based on formula (12) when it is an interval grey number sequence with a real number, due to the obstacle in transforming it into two real number sequences $S$ and $W$.

In order to take the advantage of the existing modeling method of prediction model of interval grey number proposed in literature [18], it is necessary to expand the real parameter $\phi_{m}$ in Definition 6 to an interval grey number. By doing this, a homogenous data sequence with only one type elements can be then converted from the above heterogeneous data sequences with the real parameter $\phi_{m}$. Further, we can apply the modeling methods in literature[25] to build a novel grey prediction model. The process of modeling is shown in Figure 2.

There are three steps in the process of modeling as shown in Figure 2. The study content in Step 2 actually manages to establish an interval grey number prediction model based on $\operatorname{DGM}(1,1)$ model. Such method had been discussed in literature [25]. Due to the low level of complexity, Step 2 will not be described or analyzed in this paper. The following part of this paper will mainly focus on the research on Steps 1 and 3.
3.1. Boundary Expansion of Real Parameters. Assume a real parameter $\phi_{m}$ is also an interval grey number with upper limit $a_{m}$ and lower limit $b_{m}$. The expansion of real number $\phi_{m}$ into corresponding interval grey number could be achieved by simulation process of calculating and deducing the values of $a_{m}$ and $b_{m}$. We use $\otimes\left(t_{m}\right) \in\left[a_{m}, b_{m}\right]$ to denote the corresponding interval grey number. As defined in Definitions 4 and 5 , when $m=1$, the upper limit sequence of $X(\otimes)$ is $X_{a}=\left(a_{2}, a_{3}, \ldots, a_{n}\right)$; meanwhile, the lower limit sequence is $X_{b}=\left(b_{2}, b_{3}, \ldots, b_{n}\right)$. Therefore, in order to achieve the expansion of the real number $\phi_{m}$, one approach is to build antitone (reverse) grey prediction models of sequences $X_{a}$ and $X_{b}$, so that the process to simulate the limit $a_{1}$ and lower limit $b_{1}$ of interval grey number $\otimes\left(t_{1}\right)$ could be conducted. Given that sequence $X_{a}^{\leftarrow}$ is the antitone sequence of $X_{a}$, we have

$$
\begin{aligned}
X_{a} & =\left(a_{2}, a_{3}, \ldots, a_{n}\right) \Longrightarrow X_{a}^{\leftarrow} \\
& =\left(a_{(n),(1)}, a_{(n-1),(2)}, \ldots, a_{(2),(n-1)}\right) .
\end{aligned}
$$

By building the $\operatorname{DGM}(1,1)$ model of sequence $X_{a}^{\leftarrow}$, we have

$$
\widehat{a}_{(n-k),(k+1)}=\beta_{1, a, \leftarrow}^{k-1} \times\left[a_{(n),(1)} \times\left(\beta_{1, a, \leftarrow}-1\right)+\beta_{2, a, \leftarrow}\right] .
$$

Let

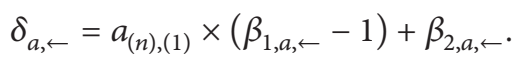

Then, formula (15) can be simplified to formula (17) as follows:

$$
\widehat{a}_{(n-k),(k+1)}=\delta_{a, \leftarrow} \times \beta_{1, a, \leftarrow}^{k-1} .
$$


According to formula (17), when $k=n-1$, we can get the value of $\widehat{a}_{(1),(n)}$; that is,

$$
\widehat{a}_{(1),(n)}=\delta_{a, \leftarrow} \times \beta_{1, a, \leftarrow}^{n-2},
$$

where $\widehat{a}_{(1),(n)}$ is the simulated value of upper limit of interval grey number $\otimes\left(t_{1}\right)$; that is, $a_{1}=\widehat{a}_{(1),(n)}$. follows:

Similarly, build the DGM $(1,1)$ model of sequence $X_{b}^{\leftarrow}$ as

$$
\widehat{b}_{(1),(n)}=\beta_{1, b, \leftarrow}^{n-2} \times\left[b_{(n),(1)} \times\left(\beta_{1, b, \leftarrow}-1\right)+\beta_{2, b, \leftarrow}\right] .
$$

Let

$$
\varphi_{b, \leftarrow}=b_{(n),(1)} \times\left(\beta_{1, b, \leftarrow}-1\right)+\beta_{2, b, \leftarrow} .
$$

Then formula (19) can be simplified to formula (21) as follows:

$$
\widehat{b}_{(1),(n)}=\varphi_{b, \leftarrow} \times \beta_{1, b, \leftarrow}^{n-2},
$$

where $\widehat{b}_{(1),(n)}$ is the simulated value of lower limit of interval grey number $\otimes\left(t_{1}\right)$; that is, $b_{1}=\widehat{b}_{(1),(n)}$.

According to formulas (19) and (21), we can get the following conclusion:

$$
\phi_{1} \Longrightarrow \otimes\left(t_{1}\right) \in\left[a_{1}, b_{1}\right] \Longrightarrow \otimes\left(t_{1}\right) \in\left[\widehat{a}_{(1),(n)}, \widehat{b}_{(1),(n)}\right] .
$$

Hence, when $m=1$, the expanded upper and lower sequences $X_{a}, X_{b}$ are

$$
\begin{aligned}
& X_{a}=\left(\widehat{a}_{(1),(n)}, a_{2}, a_{3}, \ldots, a_{n}\right), \\
& X_{b}=\left(\widehat{b}_{(1),(n)}, b_{2}, b_{3}, \ldots, b_{n}\right) .
\end{aligned}
$$

When $m=n$, the grey prediction models of sequences $X_{a}$ and $X_{b}$ can be built directly, which are used for simulating the upper limit $a_{n}$ and lower limit $b_{n}$. Next, real parameter $\phi_{n}$ can then be converted to $\otimes\left(t_{n}\right) \in\left[a_{n}, b_{n}\right]$. Consider

$$
\begin{aligned}
X_{a}=\left(a_{1}, a_{2}, \ldots, a_{n-1}\right) & \Longrightarrow \widehat{a}_{n}=\beta_{1, a}^{n-2}\left[a_{1}\left(\beta_{1, a}-1\right)+\beta_{2, a}\right], \\
X_{b}=\left(b_{1}, b_{2}, \ldots, b_{n-1}\right) & \Longrightarrow \widehat{b}_{n}=\beta_{1, b}^{n-2}\left[b_{1}\left(\beta_{1, b}-1\right)+\beta_{2, b}\right] .
\end{aligned}
$$

At the same time, provided $m=n$, the expanded upper and lower sequences $X_{a}, X_{b}$ are

$$
X_{a}=\left(a_{1}, a_{2}, \ldots, \widehat{a}_{n}\right), \quad X_{b}=\left(b_{1}, b_{2}, \ldots, \widehat{b}_{n}\right) .
$$

When $m=2,3, \ldots, n-1$, as real number $\phi_{m}$ is situated in some location of the midpiece of $X(\otimes)$, the upper and lower limit sequences $X_{a}$ and $X_{b}$ are divided into four smaller subsequences by $\phi_{m}$ (as shown in Figure 3). Due to the fact that grey prediction models are built based on small sample, whose sample size is no less than four (i.e., $n \geq 4$ ), the quantity of elements in modeling sequence is often small. Therefore, the four subsequences of $X(\otimes)$, divided by $\phi_{m}$, may not satisfy the modeling requirements of grey prediction model, shown

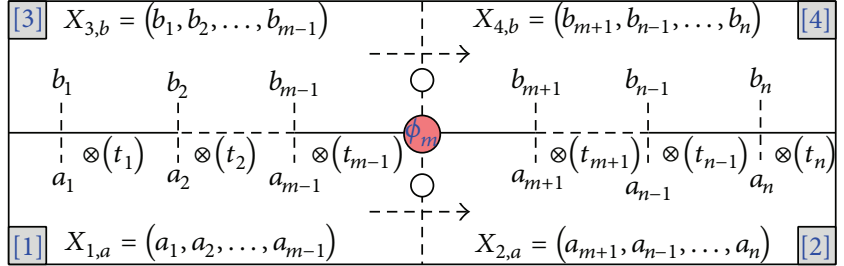

FIgURE 3: Four divided subsequences.

as $X_{1, a}, X_{2, a}, X_{3, b}$, and $X_{4, b}$ in Figure 3. Consequently, it is not feasible to expand the upper and lower limit of real parameter $\phi_{m}$ by adopting the same methodology described in the situation when $m=1$ or $m=n$. In this paper, a generation method of nonadjacent neighbor mean is proposed to tackle this task.

Assume that $\widehat{a}_{m}$ and $\widehat{b}_{m}$ are the simulated values of the upper and lower limit of real parameter $\phi_{m}$, respectively; then the upper sequence $X_{a}$ and lower sequence $X_{b}$ can be written as follows:

$$
\begin{aligned}
X_{a} & =\left(a_{1}, a_{2}, \ldots, a_{m-1}, \widehat{a}_{m}, a_{m+1}, \ldots, a_{n}\right), \\
X_{b} & =\left(b_{1}, b_{2}, \ldots, b_{m-1}, \widehat{b}_{m}, b_{m+1}, \ldots, b_{n}\right) .
\end{aligned}
$$

Then, a simple way to fill vacant data is by adopting the nonadjacent neighbor mean generation method. The calculation expressions of $\widehat{a}_{m}$ and $\widehat{b}_{m}$ are as follows:

$$
\widehat{a}_{m}=\frac{a_{m-1}+a_{m+1}}{2}, \quad \widehat{b}_{m}=\frac{b_{m-1}+b_{m+1}}{2} .
$$

Through the above studies, we have deduced a sequence with elements containing only interval grey numbers (as shown in Figure 4). Those studies create a solid foundation of building a novel grey prediction model based on DGM $(1,1)$ in Section 3.2 of this paper.

3.2. DGM $(1,1)$ Model of Interval Grey Number Sequence. As discussed in the previous session.

When $m=1$,

$$
\begin{aligned}
& X_{a}=\left(\widehat{a}_{(1),(n)}, a_{2}, a_{3}, \ldots, a_{n}\right), \\
& X_{b}=\left(\widehat{b}_{(1),(n)}, b_{2}, b_{3}, \ldots, b_{n}\right) .
\end{aligned}
$$

When $m=n$,

$$
X_{a}=\left(a_{1}, a_{2}, \ldots, \widehat{a}_{n}\right), \quad X_{b}=\left(b_{1}, b_{2}, \ldots, \widehat{b}_{n}\right) .
$$

When $m=2,3, \ldots, n-1$,

$$
\begin{aligned}
& X_{a}=\left(a_{1}, a_{2}, \ldots, a_{m-1}, \widehat{a}_{m}, a_{m+1}, \ldots, a_{n}\right), \\
& X_{b}=\left(b_{1}, b_{2}, \ldots, b_{m-1}, \widehat{b}_{m}, b_{m+1}, \ldots, b_{n}\right) .
\end{aligned}
$$

The conversion from $X_{a}$ and $X_{b}$ to the sequences $S$ and $W$ can be accomplished by adopting formula (11). Then, 


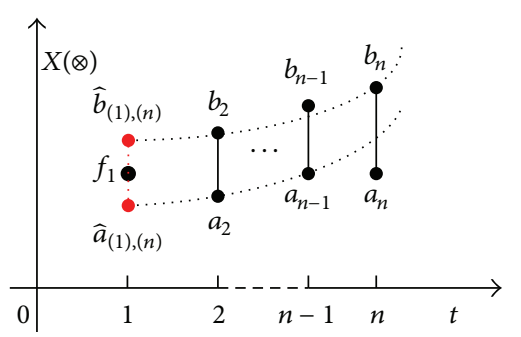

(a)

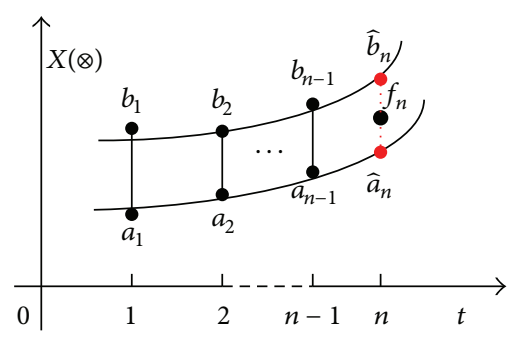

(b)

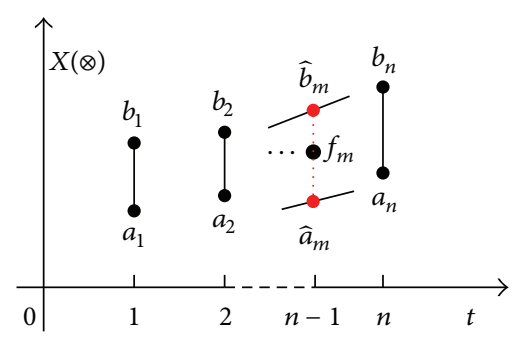

(c)

FIGURE 4: Sequence after boundary expansion of real parameter.

we employ formula (12) to build the DGM $(1,1)$ model of interval grey number sequence $X(\otimes)$. The specific derivation process is similar to the second part of this paper, and here derivation and proof will be no longer conducted.

\subsection{Prediction Model of Interval Grey Number with a Real} Parameter. The purpose of expanding boundary of real parameter is to form the complete upper and lower limit sequences. Accordingly, a prediction model can be built to forecast the boundary of unknown information. Unfortunately, it increases the inaccuracy of forecast system during the process of converting a real number into an interval grey number; in other words, known information becomes uncertain information. Hence, in order to improve the performance of the model simulation and prediction, it is crucial to develop a methodology to forecast the greatest possible value of an interval grey number based on the boundary that is forecasted from the unknown information. In this section, we will study the issue through the "kernel" of interval grey number.

A grey number's kernel is an important concept in grey system theory and also one of the basic attributes. A kernel is the most possible real number which can be used to represent the whitenization number of a grey number on the basis of full consideration of known (given) information [26]. According to literature [26], when the values' distribution of an interval grey number $\otimes\left(t_{k}\right) \in\left[a_{k}, b_{k}\right]$ is unknown, its calculation method of kernel $\widetilde{\otimes}\left(t_{k}\right)$ is as follows:

$$
\widetilde{\otimes}\left(t_{k}\right)=\frac{a_{k}+b_{k}}{2} \text {. }
$$

Since a real number can be seen as the interval grey number whose upper bound is equal to its lower bound, a real number's kernel is just itself.

Based on the above analysis, the interval grey number sequence with a real parameter can be converted into a real number sequence based on kernels according to formula (31), denoted as $\widetilde{X}(\otimes)$; that is,

$$
\widetilde{X}(\otimes)=\left(\widetilde{\otimes}\left(t_{1}\right), \widetilde{\otimes}\left(t_{2}\right), \ldots, \widetilde{\otimes}\left(t_{n}\right)\right) .
$$

Now, building a DGM $(1,1)$ model, it is achievable to define the greatest possible value of an interval grey number as follows:

$$
\widehat{\widetilde{\otimes}}\left(t_{k}\right)=\left[\left(\beta_{1, \tilde{\otimes}}-1\right) \times \widetilde{\otimes}^{(1)}\left(t_{1}\right)+\beta_{2, \tilde{\otimes}}\right] \times \beta_{1, \widetilde{\otimes}}^{k-2} .
$$

According to formulas (12) and (33), the final expression of the novel grey prediction model is as follows:

$$
\begin{gathered}
\widehat{\tilde{\otimes}}\left(t_{k}\right)=\left[\left(\beta_{1, \tilde{\otimes}}-1\right) \times \widetilde{\otimes}^{(1)}\left(t_{1}\right)+\beta_{2, \tilde{\otimes}}\right] \times \beta_{1, \tilde{\otimes}}^{k-2}, \\
\widehat{a}_{k}=\left(\left(F_{w} \times \alpha_{1}^{k-3}\left[1-\left(-\alpha_{1}^{-1}\right)^{k-2}\right]\right.\right. \\
\left.-F_{s} \times \beta_{1}^{k-3}\left[1-\left(-\beta_{1}^{-1}\right)^{k-2}\right]\right) \\
\left.\times(2)^{-1}\right)+(-1)^{k} a_{2} \\
\widehat{b}_{k}=\left(\left(F_{s} \times \beta_{1}^{k-3}\left[1-\left(-\beta_{1}^{-1}\right)^{k-2}\right]\right.\right. \\
\left.\quad+F_{w} \times \alpha_{1}^{k-3}\left[1-\left(-\alpha_{1}^{-1}\right)^{k-2}\right]\right) \\
\left.\times(2)^{-1}\right)+(-1)^{k} b_{2}
\end{gathered}
$$

and $\widehat{\otimes}\left(t_{k}\right) \in\left[\widehat{a}_{k}, \widehat{b}_{k}\right]$.

When the position of the real parameter in $X(\otimes)$ is different, the calculation methods of $F_{s}$ and $F_{w}$ in formula (34) vary accordingly, as discussed below.

When $m=1$, according to formula (11),

$$
\begin{aligned}
& s\left(t_{1}\right)=\frac{\left(\widehat{b}_{(1),(n)}-\widehat{a}_{(1),(n)}\right)+\left(b_{2}-a_{2}\right)}{2}, \\
& w\left(t_{1}\right)=\frac{\left(\widehat{a}_{(1),(n)}+\widehat{b}_{(1),(n)}\right)+\left(a_{2}+b_{2}\right)}{4} .
\end{aligned}
$$

Then,

$$
\begin{aligned}
F_{s} & =\frac{2\left[s\left(t_{1}\right) \times\left(\beta_{1}-1\right)+\beta_{2}\right]}{1+\beta_{1}^{-1}} \\
& =\frac{\left[\left(\widehat{b}_{(1),(n)}-\widehat{a}_{(1),(n)}\right)+\left(b_{2}-a_{2}\right)\right] \times\left(\beta_{1}-1\right)+2 \beta_{2}}{1+\beta_{1}^{-1}}, \\
F_{w} & =\frac{4\left[w\left(t_{1}\right) \times\left(\alpha_{1}-1\right)+\alpha_{2}\right]}{1+\alpha_{1}^{-1}} \\
& =\frac{\left[\left(\widehat{a}_{(1),(n)}+\widehat{b}_{(1),(n)}\right)+\left(a_{2}+b_{2}\right)\right] \times\left(\alpha_{1}-1\right)+4 \alpha_{2}}{1+\alpha_{1}^{-1}} .
\end{aligned}
$$


When $m=2,3, \ldots, n$, the calculation methods of $F_{s}$ and $F_{w}$ are the same as before; that is,

$$
F_{s}=\frac{\left[\left(b_{1}-a_{1}\right)+\left(b_{2}-a_{2}\right)\right] \times\left(\beta_{1}-1\right)+2 \beta_{2}}{1+\beta_{1}^{-1}},
$$

$$
F_{w}=\frac{\left[\left(a_{1}+b_{1}\right)+\left(a_{2}+b_{2}\right)\right] \times\left(\alpha_{1}-1\right)+4 \alpha_{2}}{1+\alpha_{1}^{-1}} .
$$

According to the above discussion, the final form of the novel model is described as follows:

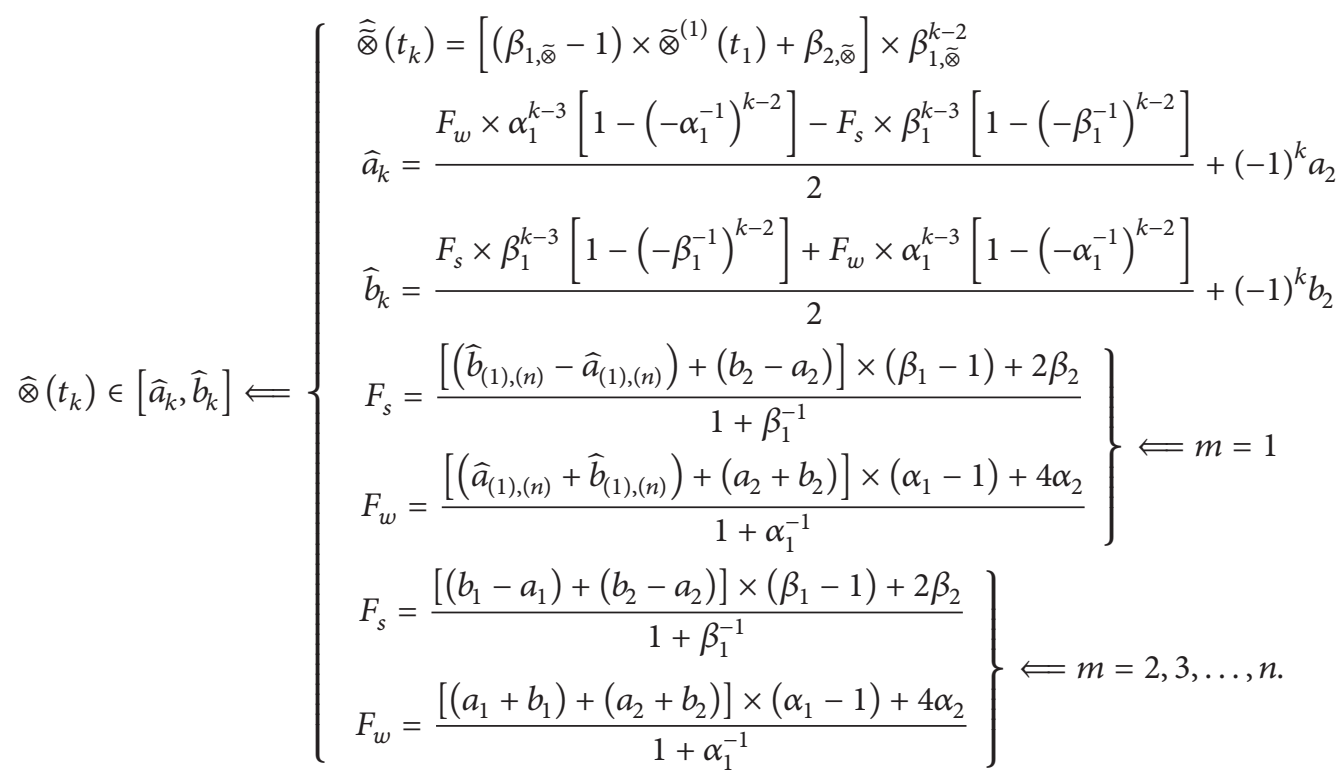

Formula (38) is called the prediction model of interval grey number with a real number $\phi_{m}$ based on DGM $(1,1)$ model, $\operatorname{IGRM}\left(1,1, \phi_{m}\right)$ model for short.

\section{Relations between IGNPM $\left(1,1, \phi_{m}\right)$ Model and Other Models}

In this section, the relations between IGRM $\left(1,1, \phi_{m}\right)$, IGM $(1,1)$ (which is short for "interval grey number prediction model"), and DGM $(1,1)$ model will be discussed.

The modeling object of DGM $(1,1)$ is a real number sequence; on the other hand, the modeling object of IGRM $\left(1,1, \phi_{m}\right)$ is an interval grey number sequence with a real number. With the growth of supplementary information regarding the grey system, when every interval grey number in IGRM $\left(1,1, \phi_{m}\right)$ becomes real numbers, in other words, when the interval grey number in IGRM $\left(1,1, \phi_{m}\right)$ 's lower limit and its corresponding upper limit overlap on one point, the model can only be built from the kernel sequence of the area sequence $S=\left(S\left(t_{1}\right), S\left(t_{2}\right), \ldots, S\left(t_{n-1}\right)\right)=(0,0, \ldots, 0)$ based on formula (33), instead of being deduced from formula (19). In fact, such model is a traditional DGM $(1,1)$ model. Therefore, the GRM $\left(1,1, \phi_{m}\right)$ is the elevation and further development based on DGM $(1,1)$ model. In addition, the relationship between them has both general and special aspect.

Similarly, when the real parameter $\phi_{m}$ in $\operatorname{IGRM}\left(1,1, \phi_{m}\right)$ is extended into an interval grey number, $\operatorname{IGRM}\left(1,1, \phi_{m}\right)$ can be seen as a standard interval grey prediction model, which is structured as same as the DGM $(1,1)$ model in formula (12). The relationship among them is shown in Figure 5.

\section{Case Study}

Persistent organic pollutants, abbreviated POPs, are toxic chemicals that adversely affect human health and the environment worldwide. They remain for long periods of time in the environment and can accumulate and pass from one species to the next through the food chain [27]. Currently, twenty-two POPs have been included in "Stockholm Convention on POPs," and twelve of them belong to organochlorine pesticides (OCPs). In recent years, the studies on OCPs have become the hot issues in the field of environmental chemistry. In particular, dichlorodiphenyltrichloroethane (DDT) is one of the common pollutants in the OCPs, which has a relative long history of usage and high accumulative production in China. In this paper, IGRM $\left(1,1, \phi_{m}\right)$ model will be used to forecast the trend of alteration in the concentration of DDT. For instance, Table 1 lists the assumed concentration values of DDT in the atmosphere of a certain city in southern China in different time intervals.

Data Specification. The monitoring data in Table 1 is not recorded from certain monitoring time point, but several continuous time periods. In addition, each continuous time period is then divided into several monitoring time points due to the fact that the value of DDT concentration in 


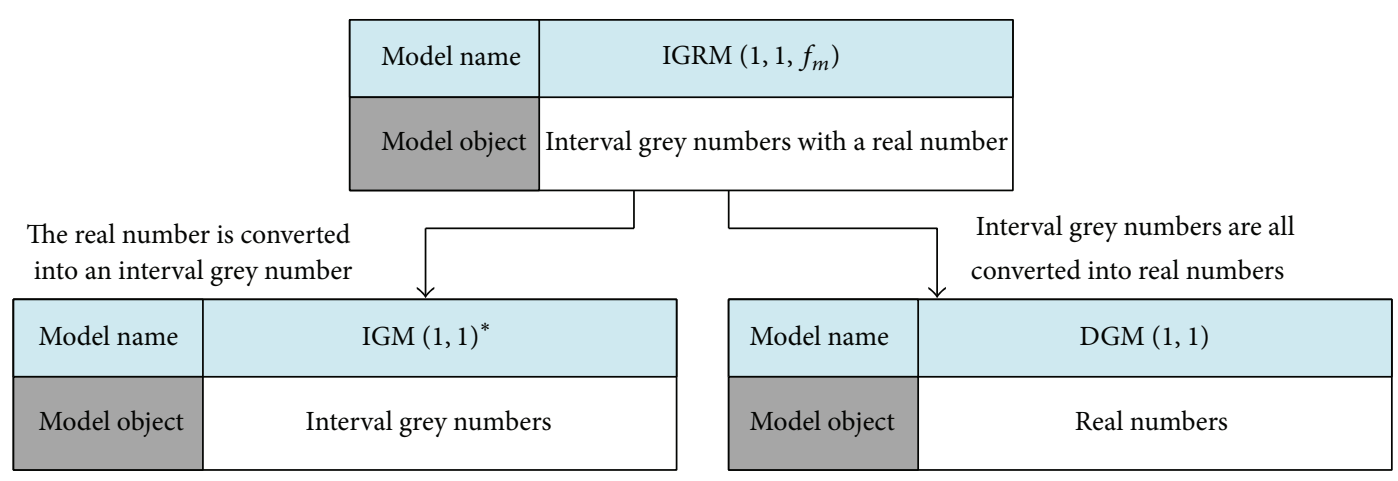

Note: $\operatorname{IGM}(1,1)^{*}$ is short for the interval grey number prediction model.

FIgURE 5: Relationships among $\operatorname{IGRM}\left(1,1, \phi_{m}\right)$, IGM $(1,1)$, and $\operatorname{DGM}(1,1)$.

TABLE 1: Concentration of DDT in atmosphere of a certain city in southern China $\left(\mathrm{ng} \cdot \mathrm{m}^{-3}\right)$.

\begin{tabular}{lc}
\hline Time range (TR) & Grey information \\
\hline TR-1 $(k=1)$ & {$[110.2,140.4]$} \\
$\operatorname{TR}-2(k=2)$ & {$[102.3,133.5]$} \\
TR-3 $(k=3)$ & {$[92.4,128.8]$} \\
TR-4 $(k=4)$ & {$[87.3,121.8]$} \\
TR-5 $(k=5)$ & {$[82.1,117.3]$} \\
TR-6 $(k=6)$ & {$[78.3,115.3]$} \\
TR-7 $(k=7)$ & {$[72.2,110.3]$} \\
TR-8 $(k=8)$ & 73.2 \\
\hline
\end{tabular}

atmosphere may not be identical at different monitoring time points, plus that we are unable to determine which monitoring value is more accurate. Hence, the value range of DDT concentration could only be composed from the lower and upper limits of collected monitoring data. This leads to the fact that the data of DDT concentration is an interval grey number (as shown in TR-1 7 in Table 1). Meanwhile, if the monitoring data collected from all monitoring time points in certain time period is a real number, then the data will be taken as the monitoring value (as shown in TR- 8 in Table 1) for this time period.

Data in Table 1 can be described as an interval grey number sequence $X(\otimes)$ with a real number; that is,

$$
\begin{array}{r}
X(\otimes)=([110.2,140.4],[102.3,133.5],[92.4,128.8], \\
{[87.3,121.8],[82.1,117.3],[78.3,115.3]}
\end{array}
$$

$[72.2,110.3], 73.2)$.

A dynamic grey prediction model of the concentration of DDT in the atmosphere is built by adopting IGRM $\left(1,1, \phi_{m}\right)$ model, and the modeling processes are described in detail in the following steps.
Step 1 (boundary expansion of real parameter). The lower limit sequence $X_{a}$ and upper limit sequence $X_{b}$ of $X(\otimes)$ are as follows according to Table 1 , respectively:

$$
\begin{gathered}
X_{a}=(110.2,102.3,92.4,87.3,82.1,78.3,72.2), \\
X_{b}=(140.4,133.5,128.8,121.8,117.3,115.3,110.3) .
\end{gathered}
$$

Then, we can build DGM $(1,1)$ models of sequences $X_{a}$ and $X_{b}$, correspondingly, as follows:

$$
\begin{aligned}
& X_{a}=\left(a_{1}, a_{2}, \ldots, a_{n-1}\right) \Longrightarrow \widehat{a}_{n}=\beta_{1, a}^{n-2}\left[a_{1}\left(\beta_{1, a}-1\right)+\beta_{2, a}\right], \\
& X_{b}=\left(b_{1}, b_{2}, \ldots, b_{n-1}\right) \Longrightarrow \widehat{b}_{n}=\beta_{1, b}^{n-2}\left[b_{1}\left(\beta_{1, b}-1\right)+\beta_{2, b}\right],
\end{aligned}
$$

$\beta_{a}=\left[\beta_{1, a}, \beta_{2, a}\right]^{T}=[0.9356,107.7420]^{T}, \quad \beta_{b}=\left[\beta_{1, b}, \beta_{2, b}\right]^{T}=$ $[0.9625,138.2729]^{T}$, the average relative error $\Delta_{a}=1.0713 \%$, $\Delta_{a}=0.7648 \%$, and the prediction values $\widehat{a}_{8}$ and $\widehat{b}_{8}$ are as follows:

$$
\begin{aligned}
& \widehat{a}_{8}=\beta_{1, a}^{8-2}\left[a_{1} \times\left(\beta_{1, a}-1\right)+\beta_{2, a}\right]=67.5 \\
& \widehat{b}_{8}=\beta_{1, b}^{8-2}\left[b_{1} \times\left(\beta_{1, b}-1\right)+\beta_{2, b}\right]=105.8
\end{aligned}
$$

Using above equations, we get $\otimes\left(t_{8}\right) \in\left[a_{8}, b_{8}\right]=[67.5,105.8]$. Hence, the expanded interval grey number sequence $X^{\prime}(\otimes)$ is as follows.

Hence, $\otimes\left(t_{8}\right) \in\left[a_{8}, b_{8}\right]=[67.5,105.8]$, the expanded interval grey number sequence $X^{\prime}(\otimes)$ is as follows

$$
X(\otimes) \Longrightarrow X^{\prime}(\otimes)=([110.2,140.4],[102.3,133.5]
$$

$[92.4,128.8],[87.3,121.8]$,

$$
\text { [82.1, 117.3], [78.3, 115.3], }
$$


Step 2 (building prediction model of interval grey number sequence $\left.X^{\prime}(\otimes)\right)$. Sequences $S$ and $W$ can be computed according to formula (11) and sequence $X^{\prime}(\otimes)$ as follows:

$$
\begin{aligned}
S & =\left(s\left(t_{1}\right), s\left(t_{2}\right), \ldots, s\left(t_{7}\right)\right) \\
& =(30.7,33.8,36.0,34.9,36.1,37.5,38.2), \\
W & =\left(w\left(t_{1}\right), w\left(t_{2}\right), \ldots, w\left(t_{7}\right)\right) \\
& =(121.6,114.3,107.6,102.1,98.3,94.0,89.0) .
\end{aligned}
$$

By building DGM $(1,1)$ of sequences $S$ and $W$, the corresponding parameters and relative errors can be achieved as follows.

Parameters of $\operatorname{DGM}(1,1)$ of sequence $S: \beta=\left[\beta_{1}\right.$, $\left.\beta_{2}\right]^{T}=[1.0222,33.4525]^{T}$ and $\Delta_{\beta}=1.3657 \%$.

Parameters of DGM $(1,1)$ of sequence $W: \alpha=\left[\alpha_{1}\right.$, $\left.\alpha_{2}\right]^{T}=[0.9525,119.3146]^{T}$ and $\Delta_{\alpha}=0.4744 \%$.

Then, parameters $F_{s}$ and $F_{w}$ of $\operatorname{IGRM}\left(1,1, \phi_{m}\right)$ can be computed as follows:

$$
\begin{aligned}
F_{s} & =\frac{\left[\left(b_{1}-a_{1}\right)+\left(b_{2}-a_{2}\right)\right] \times\left(\beta_{1}-1\right)+2 \beta_{2}}{1+\beta_{1}^{-1}} \\
& =\frac{61.4 \times(1.0222-1)+2 \times 33.4525}{1+1.0222^{-1}}=34.5, \\
F_{w} & =\frac{\left[\left(a_{1}+b_{1}\right)+\left(a_{2}+b_{2}\right)\right] \times\left(\alpha_{1}-1\right)+4 \alpha_{2}}{1+\alpha_{1}^{-1}} \\
& =\frac{486.4 \times(0.9525-1)+4 \times 119.3146}{1+0.9525^{-1}}=221.6 .
\end{aligned}
$$

Taking $F_{s}$ and $F_{w}$ into formula (38), we can get the following results:

$$
\begin{aligned}
& \widehat{a}_{k}=(\left(221.6 \times 0.9525^{k-3}\left[1-\left(-0.9525^{-1}\right)^{k-2}\right]\right. \\
&\left.-34.5 \times 1.0222^{k-3}\left[1-\left(-1.0222^{-1}\right)^{k-2}\right]\right) \\
&\left.\times(2)^{-1}\right) \longrightarrow+(-1)^{k} \times 102.3, \\
& \widehat{b}_{k}=\left(\left(34.5 \times 1.0222^{k-3}\left[1-\left(-1.0222^{-1}\right)^{k-2}\right]\right.\right. \\
&\left.\quad+221.6 \times 0.9525^{k-3}\left[1-\left(-0.9525^{-1}\right)^{k-2}\right]\right) \\
&\left.\quad \times(2)^{-1}\right) \longrightarrow+(-1)^{k} \times 133.5 .
\end{aligned}
$$

Formula (46) is the interval grey number prediction model of the concentration of DDT in the atmosphere.

Step 3 (IGRM $\left(1,1, \phi_{m}\right)$ model of the concentration of DDT in the atmosphere). First, build the $\operatorname{DGM}(1,1)$ model of "kernel" (which is the greatest possible value of interval grey number in its range) sequence. The expanded interval grey number sequence $X^{\prime}(\otimes)$ is as follows:

$$
\begin{aligned}
X(\otimes) \Longrightarrow X^{\prime}(\otimes)= & ([110.2,140.4],[102.3,133.5], \\
& {[92.4,128.8],[87.3,121.8], } \\
& {[82.1,117.3],[78.3,115.3], } \\
& {[72.2,110.3],[67.5,105.8]) }
\end{aligned}
$$

According to formula (31), the kernel sequence $\widetilde{X}(\otimes)$ of $X^{\prime}(\otimes)$ is as follows:

$$
\widetilde{X}(\otimes)=(125.3,117.9,110.6,104.6,98.7,96.8,91.3,73.2) .
$$

Building the DGM $(1,1)$ model of $\widetilde{X}(\otimes)$, one has

$$
\widehat{\widetilde{\otimes}}\left(t_{k}\right)=\left[\left(\beta_{1, \widetilde{\otimes}}-1\right) \times \widetilde{\otimes}^{(1)}\left(t_{1}\right)+\beta_{2, \widetilde{\otimes}}\right] \times \beta_{1, \widetilde{\otimes}}^{k-2},
$$

where the parameters of DGM $(1,1)$ of $\widetilde{X}(\otimes)$ are $\beta_{\widetilde{\otimes}}=\left[\beta_{1, \widetilde{\otimes}}\right.$, $\left.\beta_{2, \tilde{\otimes}}\right]^{T}=[0.9379,126.8616]^{T}$ and $\Delta_{\widetilde{\otimes}}=3.2818 \%$. Consider

$$
\begin{aligned}
\widehat{\widetilde{\otimes}}\left(t_{k}\right) & =\left[\left(\beta_{1, \tilde{\otimes}}-1\right) \times \widetilde{\otimes}^{(1)}\left(t_{1}\right)+\beta_{2, \tilde{\otimes}}\right] \times \beta_{1, \tilde{\otimes}}^{k-2} \\
& =119.0753 \times 0.9379^{k-2} .
\end{aligned}
$$

Further, by combining formulas (46) and (50), we have

$$
\begin{gathered}
\widehat{\widetilde{\otimes}}\left(t_{k}\right)=119.0753 \times 0.9379^{k-2}, \\
\widehat{a}_{k}=\left(\left(221.6 \times 0.9525^{k-3}\left[1-\left(-0.9525^{-1}\right)^{k-2}\right]\right.\right. \\
\left.-34.5 \times 1.0222^{k-3}\left[1-\left(-1.0222^{-1}\right)^{k-2}\right]\right) \\
\left.\times(2)^{-1}\right) \longrightarrow+(-1)^{k} \times 102.3, \\
\widehat{b}_{k}=\left(\left(34.5 \times 1.0222^{k-3}\left[1-\left(-1.0222^{-1}\right)^{k-2}\right]\right.\right. \\
\left.+221.6 \times 0.9525^{k-3}\left[1-\left(-0.9525^{-1}\right)^{k-2}\right]\right) \\
\left.\times(2)^{-1}\right) \longrightarrow+(-1)^{k} \times 133.5 .
\end{gathered}
$$

Formula (51) is the $\operatorname{IGRM}\left(1,1, \phi_{m}\right)$ model of the concentration of DDT in the atmosphere. It is a dynamic prediction model. Based on the value of $k$, we can forecast the range and greatest possible value of the concentration of DDT.

Step 4 (forecasting the range and greatest possible value of the concentration of DDT when $k=9,10,11)$. Forecasting is a method for speculating and acquiring information of the future, by analyzing and summarizing the historical laws of system development, on the condition that the system develops according to such historical laws. Therefore, the key factor of evaluating the quality of a model's prediction 
TABLE 2: Simulated values and errors of upper limits.

\begin{tabular}{|c|c|c|c|c|}
\hline \multirow[b]{2}{*}{ Time range (TR) } & \multicolumn{4}{|c|}{ Compared items } \\
\hline & $\begin{array}{c}\text { Original values } \\
a_{k} \\
\end{array}$ & $\begin{array}{c}\text { Simulated values } \\
\widehat{a}_{k}\end{array}$ & $\begin{array}{c}\text { Errors } \\
\varepsilon_{k}=a_{k}-\widehat{a}_{k}\end{array}$ & $\begin{array}{c}\text { Relative errors } \\
\Delta_{k}=\left|\varepsilon_{k}\right| / a_{k}\end{array}$ \\
\hline TR-1 $(k=1)$ & 110.2 & 102.8 & 7.4 & $6.7151 \%$ \\
\hline TR-2 $(k=2)$ & 102.3 & 102.3 & 0.0 & $0.0000 \%$ \\
\hline TR-3 $(k=3)$ & 92.4 & 90.7 & 1.7 & $1.8398 \%$ \\
\hline TR-4 $(k=4)$ & 87.3 & 90.8 & -3.5 & $4.0091 \%$ \\
\hline TR-5 $(k=5)$ & 82.1 & 79.7 & 2.4 & $2.9233 \%$ \\
\hline TR-6 $(k=6)$ & 78.3 & 80.2 & -1.9 & $2.4266 \%$ \\
\hline TR-7 $(k=7)$ & 72.2 & 69.5 & 2.7 & $3.7396 \%$ \\
\hline TR-8 $(k=8)$ & 67.5 & 70.5 & -3.0 & $4.4444 \%$ \\
\hline \multicolumn{4}{|c|}{ The mean relative error $\Delta_{a}=(1 / 8) \sum_{k=1}^{8} \Delta_{k}$} & $3.2622 \%$ \\
\hline
\end{tabular}

TABLE 3: Simulated values and errors of lower limits.

\begin{tabular}{|c|c|c|c|c|}
\hline \multirow[b]{2}{*}{ Time range (TR) } & \multicolumn{4}{|c|}{ Compared items } \\
\hline & $\begin{array}{c}\text { Original values } \\
b_{k}\end{array}$ & $\begin{array}{c}\text { Simulated values } \\
\widehat{b}_{k}\end{array}$ & $\begin{array}{c}\text { Errors } \\
\varepsilon_{k}=b_{k}-\widehat{b}_{k}\end{array}$ & $\begin{array}{l}\text { Relative errors } \\
\Delta_{k}=\left|\varepsilon_{k}\right| / b_{k}\end{array}$ \\
\hline TR-1 $(k=1)$ & 140.4 & 138.3 & 2.1 & $1.4957 \%$ \\
\hline TR-2 $(k=2)$ & 133.5 & 133.5 & 0.0 & $0.0000 \%$ \\
\hline TR-3 $(k=3)$ & 128.8 & 127.8 & 1.0 & $0.77640 \%$ \\
\hline TR-4 $(k=4)$ & 121.8 & 123.5 & -1.7 & $1.3957 \%$ \\
\hline TR-5 $(k=5)$ & 117.3 & 118.2 & -0.9 & $0.7673 \%$ \\
\hline TR-6 $(k=6)$ & 115.3 & 114.5 & 0.8 & $0.6938 \%$ \\
\hline TR-7 $(k=7)$ & 110.3 & 109.7 & 0.6 & $0.5440 \%$ \\
\hline TR-8 $(k=8)$ & 105.8 & 106.4 & -0.6 & $0.5671 \%$ \\
\hline \multicolumn{4}{|c|}{ The mean relative error $\Delta_{b}=(1 / 8) \sum_{k=1}^{8} \Delta_{k}$} & $0.7800 \%$ \\
\hline
\end{tabular}

function lies in whether a model can predict the development regulation of system effectively. As a result, it is of necessity to evaluate the precision of the simulation, prior to adopting the model for the purpose of predicting the development trend. Such model can be employed only if it satisfies the testing requirements. In general, the average relative error check is the most common test method for grey prediction models.

In this paper, the errors of the model simulation are analyzed from three different perspectives, presented in formula (51). They are lower limit sequence, upper limit sequence, and kernel sequence, respectively. The evaluation result is shown in Tables 2, 3 and 4.

The comparison between original and simulated values is shown in Figure 6.

Finally, the synthetic simulated error $\Delta$ of $\operatorname{IGRM}\left(1,1, \phi_{m}\right)$ model of the concentration of DDT in the atmosphere can be calculated as follows:

$$
\Delta=\frac{1}{3}\left(\Delta_{a}+\Delta_{b}+\Delta_{k e}\right)=2.2643 \%
$$

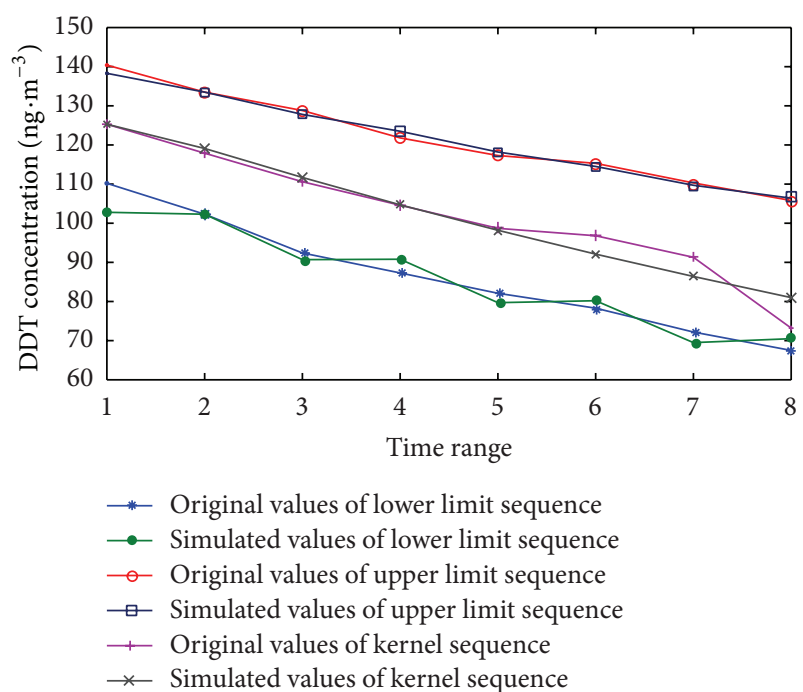

FIGURE 6: Comparison of original and simulated values. 
TABLE 4: Simulated values and errors of kernels.

\begin{tabular}{lcccc}
\hline \multirow{2}{*}{ Time range (TR) } & Original values & Simulated values & Errors & Relative errors \\
& $\tilde{\otimes}\left(t_{k}\right)$ & $\tilde{\widetilde{\otimes}}\left(t_{k}\right)$ & $\varepsilon_{k}=\tilde{\otimes}\left(t_{k}\right)-\tilde{\widetilde{\otimes}}\left(t_{k}\right)$ & $\Delta_{k}=\left|\varepsilon_{k}\right| / \widetilde{\otimes}\left(t_{k}\right)$ \\
\hline TR-1 $(k=1)$ & 125.3 & 125.3 & 0.0 & $0.0000 \%$ \\
TR-2 $(k=2)$ & 117.9 & 119.1 & -1.2 & $1.0178 \%$ \\
TR-3 $(k=3)$ & 110.6 & 111.7 & -1.1 & $0.0099 \%$ \\
TR-4 $(k=4)$ & 104.6 & 104.7 & -0.1 & $0.0956 \%$ \\
TR-5 $(k=5)$ & 98.7 & 98.2 & 0.5 & $0.0051 \%$ \\
TR-6 $(k=6)$ & 96.8 & 92.1 & 4.7 & $4.8554 \%$ \\
TR-7 $(k=7)$ & 91.3 & 86.4 & 4.9 & $5.3669 \%$ \\
TR-8 $(k=8)$ & 73.2 & -7.8 & $10.6558 \%$ \\
\hline \multicolumn{5}{c}{} \\
\hline
\end{tabular}

TABLE 5: Prediction values of concentration of DDT in atmosphere of a certain city in southern China $\left(\mathrm{ng} \cdot \mathrm{m}^{-3}\right)$.

\begin{tabular}{lccc}
\hline Time range (TR) & TR-9 $(k=9)$ & TR-10 $(k=10)$ & TR-11 $(k=11)$ \\
\hline Interval grey information & $\hat{\widetilde{\otimes}}\left(t_{9}\right) \in[60.2,102.1]$ & $\tilde{\widetilde{\otimes}}\left(t_{10}\right) \in[61.5,99.2]$ & $\hat{\tilde{\otimes}}\left(t_{11}\right) \in[51.7,95.3]$ \\
The greatest possible value (kernel) & $\tilde{\tilde{\otimes}}\left(t_{9}\right)=82.2$ & $\tilde{\tilde{\otimes}}\left(t_{10}\right)=78.2$ & $\tilde{\tilde{\otimes}}\left(t_{11}\right)=74.4$ \\
\hline
\end{tabular}

It is shown that the precision of IGRM $\left(1,1, \phi_{m}\right)$ model is between the first and second classes according to the level of accuracy test reference table [28]. So, this model can be used in the mid-short term prediction. Prediction values are shown in Table 5 for $k=9,10,11$.

\section{Discussion}

Although the interval grey number sequence with only one real number is studied in this paper, the modeling method is of reference and practical meaning for the scenarios when the quantity of real number is greater than $1(n>1)$. In this case, such real numbers can be expanded by adopting generation method of adjacent (or nonadjacent) neighbor mean, and then the corresponding grey prediction model could be built according to formula (38). Before modeling, the precision of simulation or prediction of IGRM $\left(1,1, \phi_{m}\right)$ model may be improved by various measures according to practical situations: (1) optimizing the initial and background values of IGRM $\left(1,1, \phi_{m}\right)$ model by ameliorating the smoothness degree of modeling sequence, (2) or combination of different methods, and so forth.

In the process of practical application, when the simulation error of IGRM $\left(1,1, \phi_{m}\right)$ model is too large to meet the accuracy requirement stipulated in the literature [28], it is not practical to employ IGRM $\left(1,1, \phi_{m}\right)$ model for forecasting purposes. Other approaches need to be investigated in order to solve the above problem.

\section{Conclusions}

Currently, the modeling objects of the grey prediction model are mainly time series data with the same data type. The existing models become impractical when the data types of the elements in the modeling sequence are variant (heterogeneous data sequence). In this paper, we proposed a new approach of a prediction modeling. First, the position of the real number in the interval grey number sequence is identified. Then, the real number is expanded into an interval grey number by either GM $(1,1)$ model or generation method of nonadjacent neighbor mean based on the identified position of the real number. Further, the grey prediction model of an interval grey number's kernel is built, based on deducing the novel grey prediction modeling method of interval grey number sequence with a real number. Subsequently, a new model, IGRM $\left(1,1, \phi_{m}\right)$, is presented. Finally, the above modeling method is employed to forecast the concentration of DDT in the atmosphere. Based on the data evaluation, the effectiveness and practicability of this model are verified.

In this paper, the research findings extend modeling objects of grey prediction models from homogenous data to heterogeneous data. They are of a positive significance for enriching and improving the theoretical system of grey prediction modeling. The next research target is how to build the grey prediction model of grey heterogeneous data sequence which contains both interval grey number and discrete grey number.

\section{Conflict of Interests}

The authors declare that there is no conflict of interests regarding the publication of this paper.

\section{Acknowledgments}

This work is supported by The National Natural Science Foundation, China (71271226 and 51375517), The Humanistic and Social Science Youth Foundation of Ministry of Education of China (11YJC630273 and 14YJAZH033), Program 
for Chongqing Innovation Team in University (KJTD201313), and Chongqing Frontier and Applied Basic Research Project (cstc2014jcyjA00024 and cstc2014jcyjA00037). The authors thank the anonymous referees for their constructive remarks that helped to improve the clarity and the completeness of this paper.

\section{References}

[1] A. M. Andrew, "Why the world is grey," Grey Systems: Theory and Application, vol. 1, no. 2, pp. 112-116, 2011.

[2] D. Ju-Long, "Control problem of grey systems," Systems \& Control Letters, vol. 1, no. 5, pp. 288-294, 1982.

[3] Z. Kong, L. F. Wang, and Z. X. Wu, "Application of fuzzy soft set in decision making problems based on grey theory," Journal of Computational and Applied Mathematics, vol. 236, no. 6, pp. 1521-1530, 2011.

[4] Q.-X. Li, S.-F. Liu, and Y. Lin, "Grey enterprise input-output analysis," Journal of Computational and Applied Mathematics, vol. 236, no. 7, pp. 1862-1875, 2012.

[5] S. F. Liu, J. Forrest, and Y. J. Yang, "A brief introduction to grey systems theory," Grey Systems: Theory and Application, vol. 2, no. 2, pp. 89-104, 2012.

[6] H. N. Zhang, A. J. Xu, and J. Cui, "Establishment of neural network prediction model for terminative temperature based on grey theory in hot metal pretreatment," Journal of Iron and Steel Research International, vol. 19, no. 6, pp. 25-29, 2012.

[7] L. C. Hsu, "Applying the grey prediction model to the global integrated circuit industry," Technology and Social Change, vol. 70, no. 6, pp. 563-574, 2003.

[8] X. W. Ren, Y. Q. Tang, J. Li, and Q. Yang, "A prediction method using grey model for cumulative plastic deformation under cyclic loads," Natural Hazards, vol. 64, no. 1, pp. 441-457, 2012.

[9] M. Mohammed, K. Watanabe, and S. Takeuchi, "Grey model for prediction of pore pressure change," Environmental Earth Sciences, vol. 60, no. 7, pp. 1523-1534, 2010.

[10] X. L. Li, Y. J. Li, and K. Zhang, "Improved grey lorecasting model of fault prediction in missile applications," Computer Simulation, vol. 27, pp. 33-36, 2010.

[11] Y. Wei and D. H. Hu, "Deficiency of the smoothness condition and its remedy," Systems Engineering-Theory \& Practice, vol. 29, no. 8, pp. 165-170, 2009.

[12] W. Z. Dai, Z. H. Wu, and A. P. Yang, "New buffer operators with variable weight based on average tempo and their optimization," Grey Systems: Theory and Application, vol. 1, no. 2, pp. 168-177, 2001.

[13] Y. G. Dang and S. F. Liu, "The GM models that $\mathrm{x}(\mathrm{n})$ be taken as initial value," The International Journal of Systems \& Cybernetics, vol. 33, no. 2, pp. 247-254, 2004.

[14] Z. X. Wang, Y. G. Dang, and S. F. Liu, "The optimization of background value in GM, $(1,1)$ model," The Journal of Grey System, vol. 10, no. 2, pp. 69-74, 2007.

[15] Z. M. Song, Z. D. Wang, and X. J. Tong, "Grey generating space on opposite accumulation," The Journal of Grey System, vol. 13, pp. 305-308, 2001.

[16] Z. X. Wang, Y. G. Dang, and L. L. Pei, "Combinatorial optimization of initial value in GM, $(1,1)$ power model," Statistics \& Information Forum, vol. 6, pp. 55-58, 2012.

[17] S. F. Liu and J. L. Deng, "The range suitable for $\operatorname{GM}(1,1)$," The Journal of Grey System, vol. 11, pp. 131-138, 1996.
[18] N.-M. Xie and S.-F. Liu, "Discrete grey forecasting model and its optimization," Applied Mathematical Modelling, vol. 33, no. 2, pp. 1173-1186, 2009.

[19] W. Z. Dai and J. F. Li, "Modeling research on non-equidistance GM(1, 1) model," Systems Engineering-Theory \& Practice, vol. 25, no. 9, pp. 89-93, 2005.

[20] B. Zeng, S. F. Liu, Z. G. Fang, and N. M. Xie, "Grey combined forecast models and its application," Chinese Journal of Management Science, vol. 17, pp. 150-155, 2009.

[21] C. G. Shen, W. M. Chen, and L. L. Pei, "Optimization of the initial conditions in unbiased grey verhulst model," Statistics \& Information Forum, vol. 5, pp. 3-6, 2011.

[22] H. Jiang and W. W. He, "Grey relational grade in local support vector regression for financial time series prediction," Expert Systems with Applications, vol. 39, no. 3, pp. 2256-2262, 2012.

[23] B. Zeng, S. F. Liu, and N. M. Xie, "Prediction model for interval grey number based on grey band and grey layer," Control and Decision, vol. 25, pp. 1585-1588, 2010.

[24] B. Zeng, S. F. Liu, and W. Meng, "Prediction model of discrete grey numbers based on kernels and areas," Control and Decision, vol. 26, no. 9, pp. 1421-1424, 2011.

[25] B. Zeng, S. F. Liu, and N. M. Xie, "Prediction model of interval grey number based on DGM, $(1,1)$," Journal of Systems Engineering and Electronics, vol. 21, no. 4, pp. 598-603, 2010.

[26] B. Zeng and S. F. Liu, "Calculation for kernel of interval grey number based on barycenter approach," Transactions of Nanjing University of Aeronautics \& Astronautics, vol. 30, no. 2, pp. 216220, 2013.

[27] X. Seguí, E. Pujolasus, S. Betrò et al., "Fuzzy model for risk assessment of persistent organic pollutants in aquatic ecosystems," Environmental Pollution, vol. 178, pp. 23-32, 2013.

[28] S. F. Liu and Y. Lin, Grey Systems Theory and Applications, Springer, Berlin, Germany, 2010. 


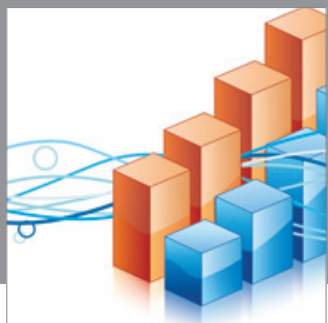

Advances in

Operations Research

mansans

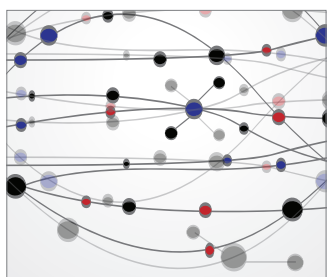

The Scientific World Journal
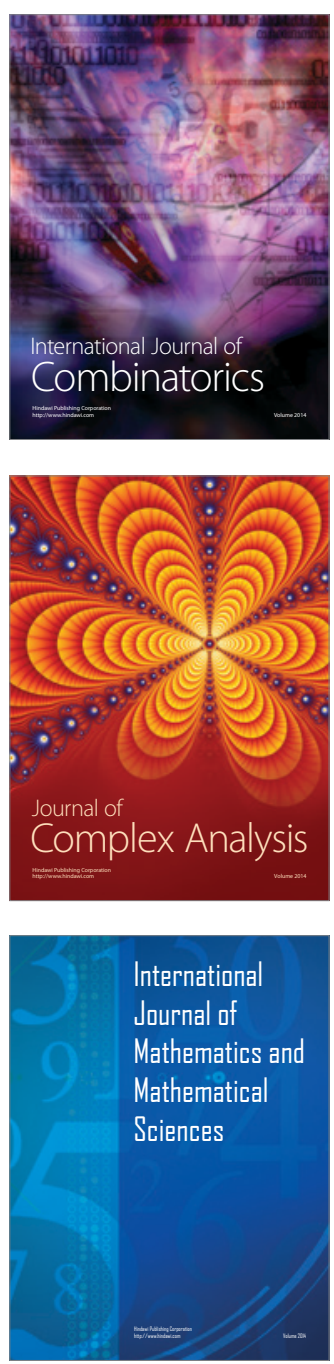
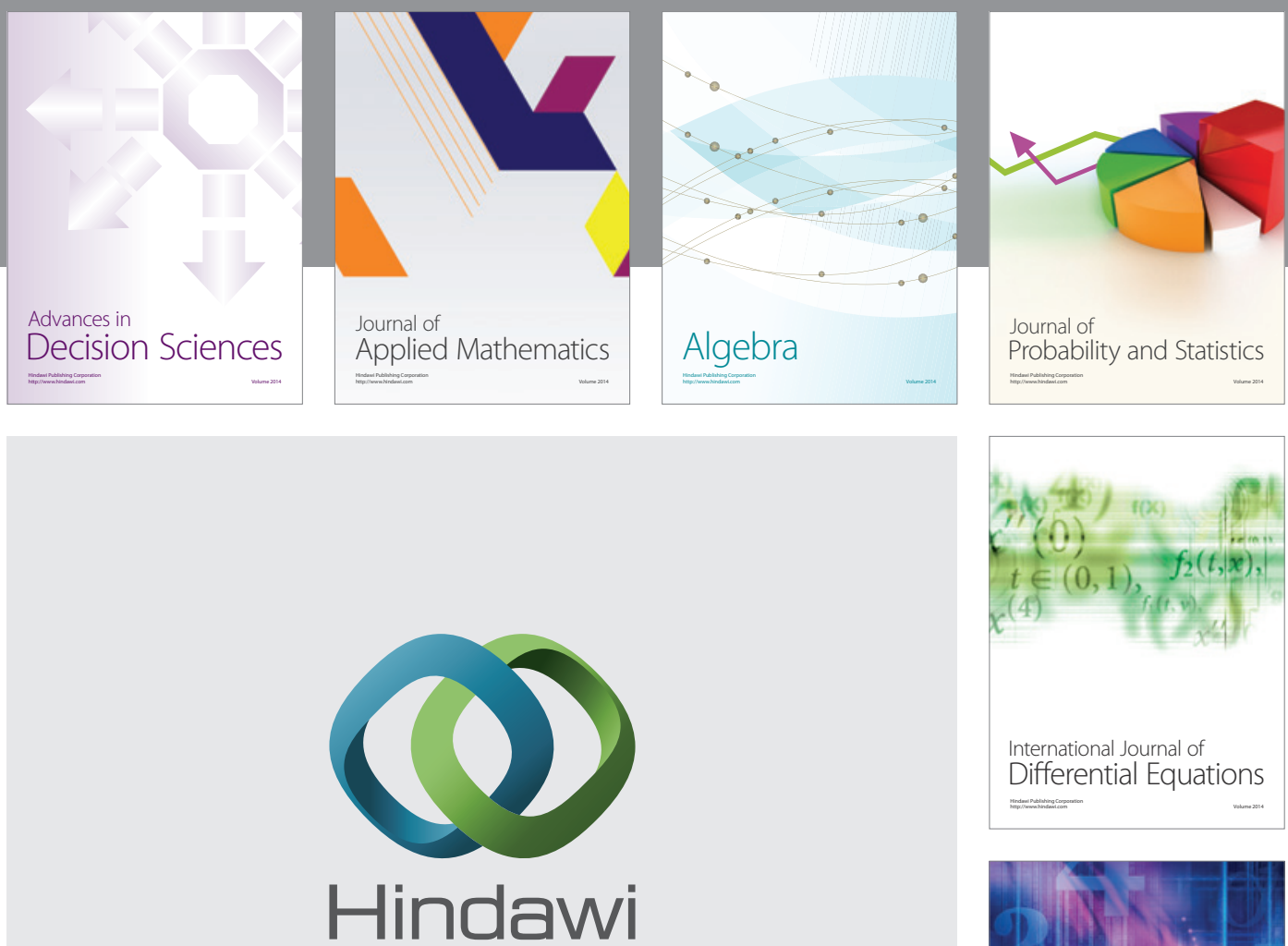

Submit your manuscripts at http://www.hindawi.com
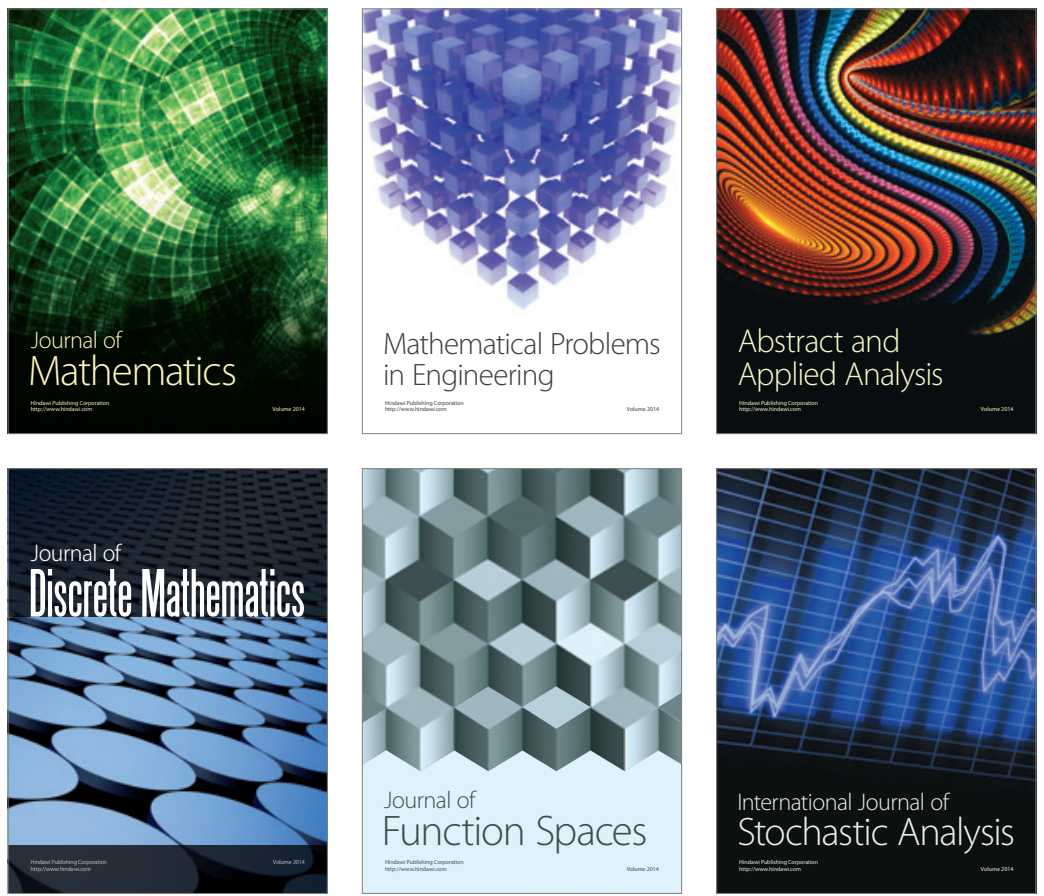

Journal of

Function Spaces

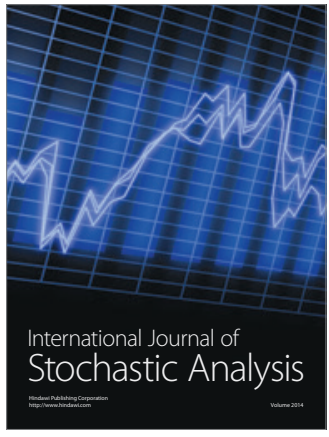

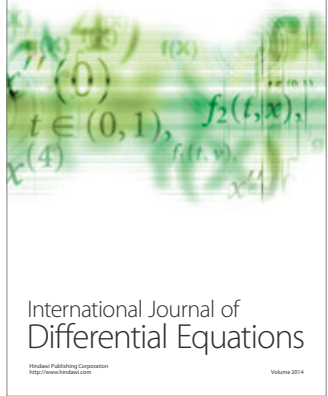
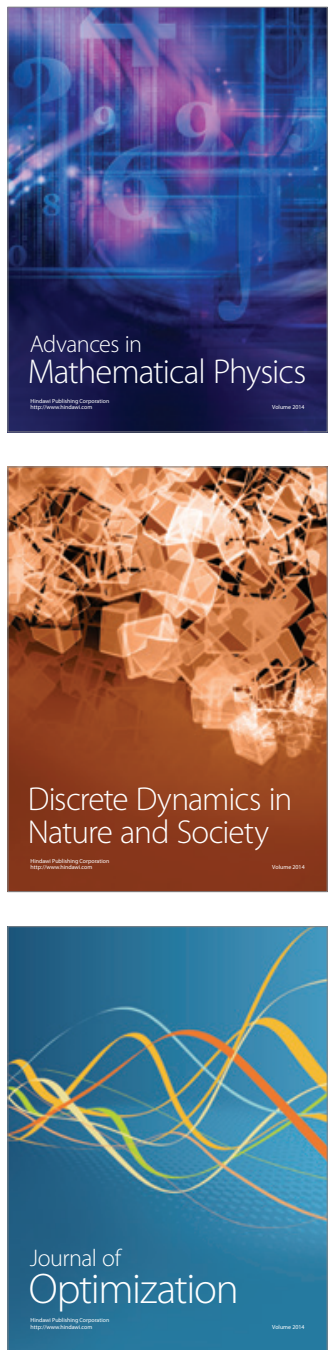